University of Wollongong

Research Online

Faculty of Engineering and Information

Faculty of Engineering and Information

Sciences - Papers: Part A

Sciences

$1-1-2012$

Wireless indoor localisation using received signal strength fingerprinting with context aware partitioning

Montserrat Ros

University of Wollongong, montse@uow.edu.au

Brendan Schoots

University of Wollongong

Matthew D'Souza

University Of Queensland, dsouza@itee.uq.edu.au

Follow this and additional works at: https://ro.uow.edu.au/eispapers

Part of the Engineering Commons, and the Science and Technology Studies Commons

Research Online is the open access institutional repository for the University of Wollongong. For further information contact the UOW Library: research-pubs@uow.edu.au 


\title{
Wireless indoor localisation using received signal strength fingerprinting with context aware partitioning
}

\author{
Abstract \\ Real-time indoor localisation tracking of people with unobtrusive, wearable sensors has valuable potential \\ for a variety of applications such as remote monitoring and tracking of aged-care patients to improve \\ their safety and other care aspects. There are no widely available or costeffective and ubiquitous wireless \\ solutions like GPS for indoor localisation which require no prior infrastructure. Indoor localisation \\ systems are available but most have difficulties operating in confined spaces or cannot localise to within \\ small distances in real-time for moving objects as required for sport and health applications.

\section{Keywords} \\ signal, context, fingerprinting, strength, received, wireless, indoor, aware, localisation, partitioning \\ Disciplines \\ Engineering | Science and Technology Studies \\ Publication Details \\ Ros, M., Schoots, B. \& D'Souza, M. 2012, 'Wireless indoor localisation using received signal strength \\ fingerprinting with context aware partitioning', Wireless Information Technology and Systems (ICWITS), \\ 2012 IEEE International Conference on, IEEE, Australia, pp. 1-4.
}




\title{
Wireless Indoor Localisation using Received Signal Strength Fingerprinting with Context Aware Partitioning
}

\author{
Montserrat Ros ${ }^{*(1)}$, Brendan Schoots ${ }^{(1)}$, and Matthew D'Souza ${ }^{(2)}$ \\ (1) University of Wollongong, Wollongong, NSW, 2000, Australia \\ (2) Australian E-Health Research Centre, CSIRO, Brisbane, QLD, 4069, Australia \\ E-mail: montse@uow.edu.au,bs949@uowmail.edu.au,matthew.d'souza@csiro.au
}

\section{Introduction}

Real-time indoor localisation tracking of people with unobtrusive, wearable sensors has valuable potential for a variety of applications such as remote monitoring and tracking of aged-care patients to improve their safety and other care aspects. There are no widely available or costeffective and ubiquitous wireless solutions like GPS for indoor localisation which require no prior infrastructure. Indoor localisation systems are available but most have difficulties operating in confined spaces or cannot localise to within small distances in real-time for moving objects as required for sport and health applications.

Current localisation techniques depend on using sensing infrastructure already present in the environment such as visual markers, wireless LAN hotpots, cellular networks or GPS satellite coverage. Indoor environments exhibit multi-path interference to Radio Frequency (RF) wireless technologies because of the presence of physical obstacles such as metal beams or walls. Hence this causes outdoor RF based localisation technologies such as GPS to function inaccurately indoors because of signal degradation. RF localisation methods such as Received Signal Strength Indicator (RSSI) or Time of Arrival also experience inaccuracies and reliability issues when operating indoors. To overcome this, we developed a Fingerprint Context Aware Partitioning (FCAP) tracking model for tracking people within a building.

The FCAP tracking model used a form of RSSI fingerprinting. Fingerprinting is a form of landmark localisation that uses distinct RSSI signatures to estimate a position. Current localisation techniques using fingerprinting can be affected by the physical layout of the indoor environment The FCAP tracking model extends fingerprinting by using context-aware information, such as a building floorplan. The use of context aware information allowed the FCAP model to improve the reliability of the position accuracy, while depending on varying fingerprint granularity.

\section{Background Literature}

Receive Signal Strength Indicators (RSSI) are used for indoor and outdoor localisation, as outlined by Seco et al [1]. The most common RSSI localisation techniques are RSSI Fingerprinting, RSSI triangulation and trilateration. RSSI Fingerprinting identifies specific positions with RSSI values, while RSSI triangulation and trilateration associate RSSI with distance or angular trajectory between receiver and known transmitter positions in order to localise. 
Hightower et al [2] describe the Placelab geophysical location system which users can determine their position in an urban environment. Placelab was an RSSI Fingerprinting technique that used wireless LAN hotspots and GSM broadcast towers to determine a user's position. The Placelab software used a database of known Wireless LAN hotspots and GSM broadcast towers. Localisation accuracy is stated as being less then GPS, with $20-25 \mathrm{~m}$ using Wireless LAN and $100 \mathrm{~m}$ to $150 \mathrm{~m}$ for GSM broadcast towers.

\section{Wireless Indoor Localisation Network Overview}

The FCAP tracking model was implemented with a ZigBee/802.15.4 wireless communications network. This was due to the capability of Zigbee as a low data rate wireless communications protocol that can operate on devices with limited computing or power resources and cater for large networks of active devices [3]. The use of Zigbee for home automation and smart metering applications allowed for a more dense network of wireless nodes in a confined area, rather than WLAN, which can be used more effectively for localisation. Other Supported Zigbee features include Mesh Networking, 64-bit address, data rates: 20kbps to 250kbps and simple application profiles. Zigbee operates in the unlicensed ISM $2.4 \mathrm{GHz}$ or $915 \mathrm{MHz}$ frequency band [3].

The wireless indoor localisation network as seen in Figure 1 consisted of three types of nodes: coordinator, reference and mobile. Mobile nodes are carried by users to determine their current location. The reference nodes are used to determine a mobile node's position. The server connected to the coordinator node displays the current positions of the mobile nodes on a building floorplan.

\section{Fingerprint Context Aware Tracking Process}

The FCAP tracking model estimated a person's position in using RSSI Fingerprinting. The RSSI fingerprints are a set of signal strength signatures that are unique to specific locations. Fingerprints can determine the likely position of the mobile by estimating which are the nearest reference nodes. The use of fingerprints can also be unreliable due to distortions of measuring RSSI by not using any location information. The integration of context-aware information for Fingerprint partitioning enables a more reliable means of determining the nearest valid reference nodes.

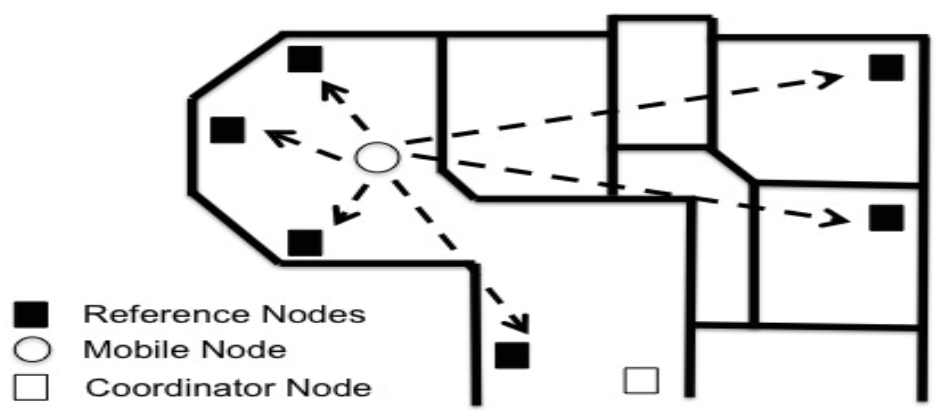

Figure 1: Wireless Indoor Localisation Network Overview 


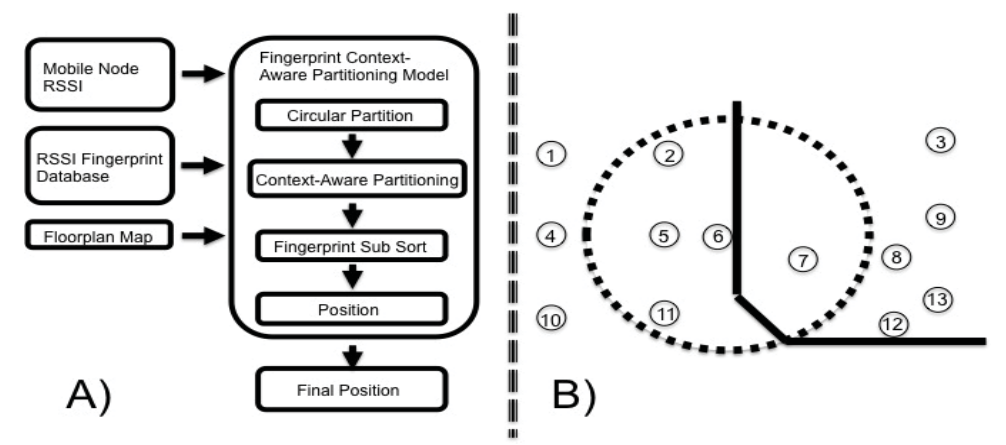

Figure 2: Overview of Fingerprint Context Aware Tracking Process

The FCAP model, seen in Figure 2 A), used the mobile node's RSSI values, RSSI fingerprint database and building floorplan features are used by the FCAP model. First the FCAP model estimates the likely position of the mobile node using circle partitioning. This estimate used context-aware information provided by the floorplan and subsampling of the RSSI values to refine the likely position. Finally, multilateration is used to estimate the mobile node's position.

\section{- Circular and Context Aware Partitioning}

The circular partitioning process is used to determine the mobile node's nearest reference nodes within a fixed radius. The reference node with the highest RSSI fingerprint is used as the center of a circular area to partition the reference nodes within the valid area. The context-information of the building floorplan is then used to reduce which number of RSSI fingerprints that are within the valid area. The floorplan consisted regions mapped with the probability of likely RSSI fingerprints available. Position validity was approximated by detecting if the mobile node's track had to move through a wall. An example of the circular partitioning process can be seen in Figure 2 B), where the large circle represents the partition encompassing the reference node 5, and the actual mobile node's location. The valid fingerprint set can be reduced to the four fingerprints within the shaded circle.

\section{- Multilateration}

Once the Fingerprints have been determined, multilateration is used to calculate the mobile node's position. The multilateration process used the least squares algorithm to determine the position of the mobile node.

\section{Evaluation}

The FCAP tracking model was tested in an indoor environment shown in Figure 3 A) and B). The placement of the reference nodes can affect the localisation accuracy using RSSI fingerprints. We tested the FCAP model by having a user walk a known path whilst carrying a mobile node. Two reference node placement layouts were used to test the FCAP model: Layout 1 and 2. Layout 1 consisted of reference nodes placed on along the outer walls (cover area with multiple reference nodes), while layout 2 consisted of reference nodes placed along the inner walls and did not confine the movement of the mobile node to areas covered by multiple reference nodes. The environment used for testing, showed signs of typical indoor RF interference. Figure 3 A) shows the localisation by multilateration only. Figure 3 B) shows the 


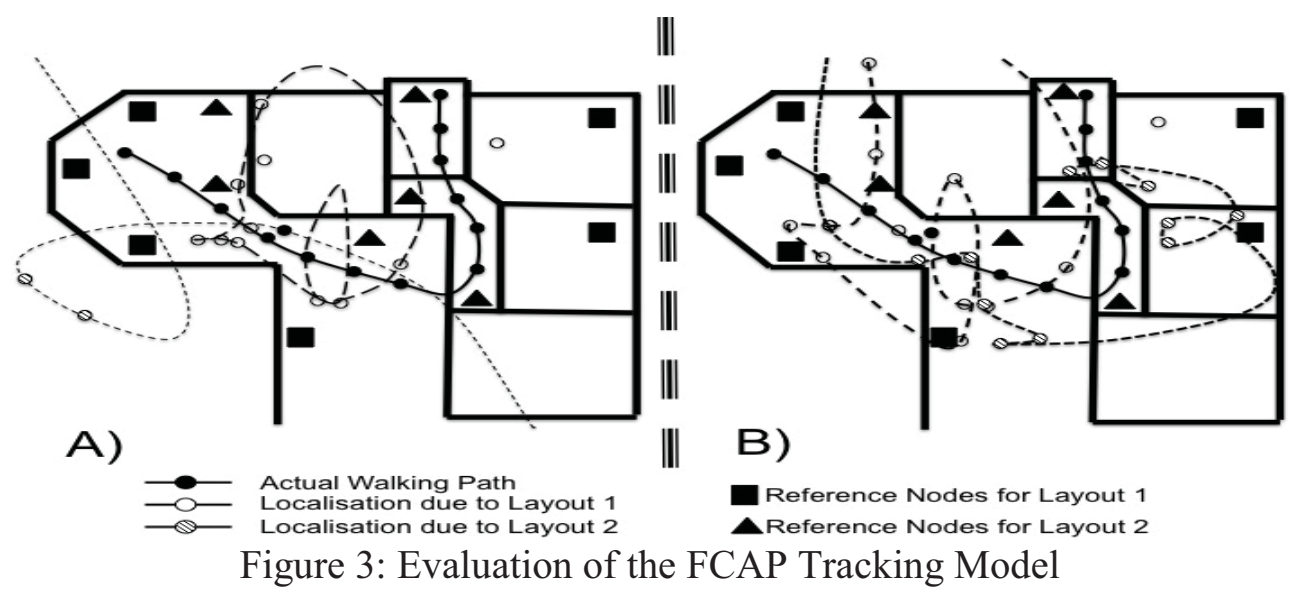

localisation using the FCAP model. Table 1 shows the average error and standard deviation measured for both layouts. While the average error was similar for multilateration and the FCAP model for layout 1, the FCAP model had less error than the multilateration results for layout 2.

Table 1: Average Error and Standard Deviation for Layout 1 and Layout 2

\begin{tabular}{|l|l|l|l|l|}
\hline Layout & Layout 1 & Layout 2 & Layout 1 (FCAP) & Layout 2 (FCAP) \\
\hline Average Error $(\mathrm{m})$ & 2.57 & 14.29 & 3.16 & 4.48 \\
\hline Standard Deviation $(\mathrm{m})$ & 1.62 & 24.54 & 2.1 & 4.96 \\
\hline
\end{tabular}

\section{Conclusion and Further Work}

We presented a wireless indoor localisation system that tracked users in an indoor environment, using the FCAP tracking model. The FCAP model used RSSI fingerprinting combined with context-aware information, such as a building floorplan. The use of context aware information allowed the FCAP model to improve position accuracy. We evaluated and compared the FCAP model to conventional multilateration. The FCAP model performed better than multilateration, where the layout of the wireless indoor localisation network did not restrict the position of the mobile node to areas covered by multiple reference nodes. This was advantageous by not restricting the placement of the reference nodes. Further work involves investigating the use of multiple operating mobile nodes with the FCAP model and how 3-dimensional localisation can be achieved with context awareness of the surrounding environment.

\section{References:}

[1] F. Seco, A. R. Jimenez, C. Prieto, J. Roa, and K. Koutsou, "A survey of mathematical methods for indoor localization," in Proceedings of the 6th IEEE International Symposium on Intelligent Signal Processing (WISP '09), pp. 9-14, Madrid, Spain, 2009.

[2] J. Hightower, A. LaMarca, and I. Smith, "Practical lessons from place lab," IEEE Pervasive Computing, vol. 5, no. 3, Article ID 1673364, pp. 32-39, 2006.

[3] ZigBee Alliance, ZigBee Specification, 2006, http://www.zigbee.org/ 